\section{Tribune libre Freies Forum}

\section{Traitement du psoriasis par des médicaments biologiques}

\author{
S. Kuenzli, Genève
}

Le 5 juin 2004 a eu lieu à Lucerne le symposium «Current Aspects of Psoriasis» organisé par Serono Pharma Suisse, au cours duquel plusieurs experts ont rapporté les plus récentes découvertes sur la pathogenèse et le traitement du psoriasis. Le principal sujet abordé dans ce colloque était l'efficacité et la tolérabilité des médicaments immunomodulateurs dits «biologiques», qui, d'après le Prof. Jean-Hilaire Saurat, président de la séance, vont contribuer à une amélioration décisive du traitement du psoriasis.

\section{Diminution importante de la qualité de la vie}

Le Prof. Alan Menter, Dallas (USA), déclare que le psoriasis s'accompagne d'une morbidité psychosociale considérable et d'une perte sensible de la qualité de la vie du patient, raison pour laquelle cette maladie doit être considérée comme un problème de santé sérieux qui nécessite un traitement conséquent.

Dans le concept stratégique traditionnel tel qu'il a prévalu jusqu'ici, le traitement du psoriasis se fait selon une approche progressive qui consiste à administrer initialement des médicaments topiques pour passer successivement à la photothérapie, puis au traitement systémique dans les cas où l'efficacité est insuffisante. Le traitement systémique du psoriasis modéré à sévère fait appel à des rétinoïdes, à des corticostéroïdes, au méthotrexate et à la cyclosporine, et ces derniers ne possèdent souvent qu'une efficacité limitée. Ainsi, une enquête auprès de patients psoriasiques sévères a montré que le traitement systémique était ressenti comme frustrant dans $78 \%$ des cas et pas assez agressif dans $32 \%$ des cas. Outre son efficacité insuffisante, l'utilité du traitement systémique au long cours traditionnel est souvent limitée par une toxicité considérable liée au manque de spécificité des médicaments utilisés. Aussi le traitement est-il généralement interrompu après disparition des symptômes, mais la plupart des patients ne tardent pas à rechuter. Il existe par conséquent un besoin urgent de nouvelles stratégies de contrôle à long terme du psoriasis. Or, on dispose maintenant, depuis quelque temps, de molécules biologiques qui laissent entrevoir un progrès décisif dans le traitement du psoriasis modéré à sévère.

Cet élargissement de l'arsenal thérapeutique devrait permettre de remplacer la stratégie progressive, appliquée jusqu'ici, par un traitement individualisé qui pourra être ajusté à chaque patient en fonction du degré de sévérité et de l'extension de sa maladie et selon ses besoins subjectifs.

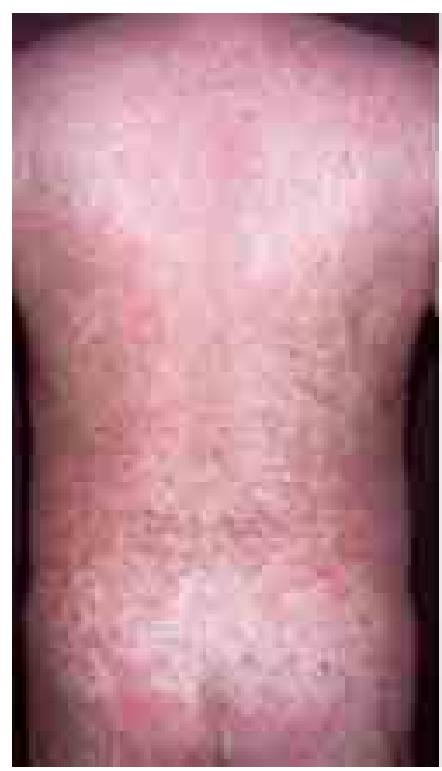

Fig. 1. a Raptiva monothérapie: mois 0 .

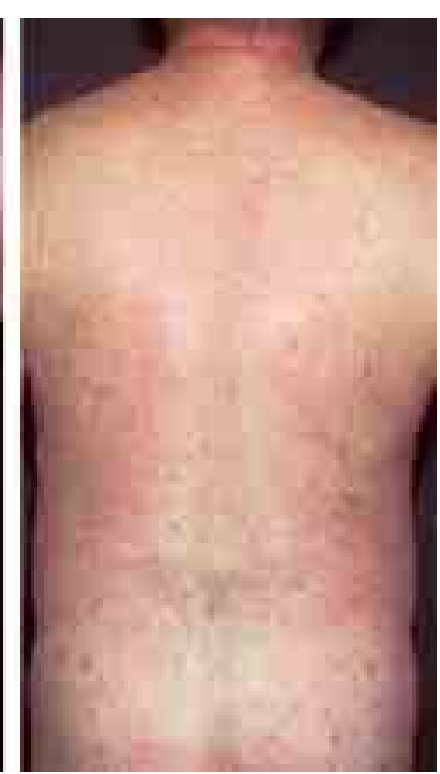

b Mois 2 .

\section{Immunophysiopathologie du psoriasis}

Le PD Dr Nikhil Yawalkar, de Berne (Suisse), a expliqué que le psoriasis est une maladie multifactorielle, c'est-à-dire qu'elle peut être déclenchée par un grand nombre de facteurs chez des personnes génétiquement prédisposées. Ces facteurs comprennent les infections bactériennes, la prise de certains médicaments, la consommation immodérée d'alcool ou le stress psychique. D'après notre compréhension actuelle de sa pathogenèse, le psoriasis est probablement dû à une réaction auto-immune où intervient un dysfonctionnement des lymphocytes T. Dans cette réaction auto-immune, un antigène non encore précisément identifié est incorporé par certaines cellules de la peau qui présentent ensuite cet antigène à leur surface. Ces cellules présentatrices d'antigène migrent ensuite le long des voies lymphatiques de la peau vers les ganglions lymphatiques, où l'antigène présenté est reconnu par les lymphocytes T par l'entremise du «complexe majeur d'histocompatibilité» et des «récepteurs de cellules T». La présentation d'antigène et l'interaction qui en résulte entre des molécules de surface spécifiques des cellules impliquées entraîne une activation et une prolifération des lymphocytes $\mathrm{T}$ qui retournent vers le derme et l'épiderme par la circulation sanguine. Une réactivation antigène spécifique de ces lymphocytes $T$ effecteurs et à mémoire se produit dans la peau, entraînant une libération d'interféron $\alpha$ et du facteur de nécrose tumorale $\alpha$. Ces cytokines libérées provoquent une réaction inflammatoire ainsi qu'une prolifération et une différenciation anormales des kératinocytes, ce qui aboutit à la formation des plaques psoriasiques caractéristiques (fig. 1). L'amélioration des connaissances sur la physiopathologie du psoriasis a conduit au développement de molécules biologiques qui inhibent l'activation des lymphocytes $T$ ou détruisent de manière sélective les lymphocytes $\mathrm{T}$ activés, ou encore les bloquent de manière ciblée. 


\section{Nouvelles stratégies thérapeutiques}

Le Prof. Christopher Griffiths, de Manchester (GB), rapporte que sont parus entre-temps les résultats de plusieurs études cliniques qui ont examiné l'utilité thérapeutique de quatre médicaments biologiques distincts dans le traitement du psoriasis. La protéine de fusion aléfacept élimine les lymphocytes $\mathrm{T}$ effecteurs/mémoire. L'anticorps monoclonal humanisé éfalizumab inhibe l'activation, la migration et la réactivation des lymphocytes $\mathrm{T}$.

En plus des deux médicaments agissant sur les lymphocytes $\mathrm{T}$, des études cliniques ont examiné deux autres molécules biologiques (l'étanercept et l'infliximab) dont l'efficacité repose sur le blocage du facteur de nécrose tumorale $\alpha$.

Les résultats des études cliniques réalisées à ce jour semblent indiquer que les biologiques permettent un contrôle efficace du psoriasis, de sorte que ces préparations offrent un précieux enrichissement des possibilités thérapeutiques, en particulier dans les formes sévères de la maladie. Le psoriasis étant une maladie chronique qui évolue par poussées, le but du traitement est non seulement d'atteindre une rémission, mais de la maintenir à long terme.

\section{Expérience à long terme avec l'éfalizumab}

Le Dr Kim Papp, Ontario (Canada), a signalé qu'à ce jour, plus de 2700 patients psoriasiques ont été traités par l'éfalizumab sur une durée jusqu'à 2 ans dans le cadre d'études cliniques. Les données actuellement disponibles sur l'éfalizumab portent sur des durées de traitement de 12 et 24 semaines et de 24 mois (tab. 1).

Le traitement par l'éfalizumab s'est avéré bien toléré et on n'a pas constaté d'augmentation de l'incidence d'infections ou d'atteintes malignes chez les patients du groupe éfalizumab par rapport au groupe placébo, y compris lors du traitement prolongé sur 24 mois.

Le développement des médicaments biologiques a permis de faire un progrès décisif dans le traitement du psoriasis, ces médicaments produisant souvent un net recul des symptômes même chez des patients atteints de formes sévères de la maladie. Les résultats des premières études au long cours indiquent d'autre part qu'en plus d'une induction rapide de la rémission, les médicaments biologiques permettent aussi un contrôle durable des symptômes.

\begin{tabular}{|c|c|c|c|}
\hline & 12 semaines $^{1}$ & 24 semaines $^{2}$ & 24 mois $^{3}$ \\
\hline PASI 50 & $59 \%$ & $67 \%$ & \\
\hline PASI 75 & $27 \%$ & $44 \%$ & $68 \%$ \\
\hline PASI 90 & & & $36 \%$ \\
\hline
\end{tabular}

${ }^{1}$ Contrôle par placébo.

2 En ouvert.

${ }^{3}$ En ouvert, analyse selon traitement effectif («as treated»).
Dr Stéphane Kuenzli

Hôpital cantonal

Clinique de dermatologie

Rue Micheli-du-Crest 24

CH-1205 Genève

Tél. 0223729423

Fax 0223729470

\section{Psoriasis- behandlung mit biologischen Wirkstoffen}

\section{S. Kuenzli, Genf}

Am 5. Juni 2004 fand in Luzern das von Serono Pharma Schweiz organisierte Symposium «Current Aspects of Psoriasis» statt, an dem verschiedene Experten über die neusten Erkenntnisse zur Pathogenese und Behandlung der Psoriasis berichteten. Als zentrales Thema dieser Veranstaltung wurde auf die Wirksamkeit und Verträglichkeit von immunmodulierenden Präparaten, den sogenannten Biologika, eingegangen, die nach Chairman Prof. Jean-Hilaire Saurat zu einer entscheidenden Verbesserung der Behandlung der Psoriasis beitragen dürften.

\section{Starke Beeinträchtigung der Lebensqualität}

Laut Prof. Alan Menter, Dallas (USA), ist Psoriasis mit einer erheblichen psychosozialen Morbidität und einer beträchtlichen Einbusse an Lebensqualität verbunden, weshalb die Psoriasis als schwerwiegendes Gesundheitsproblem zu betrachten ist und eine konsequente Therapie erfordert.

Gemäss dem bisher angewendeten traditionellen Konzept erfolgt die Behandlung der Psoriasis nach einem stufenweisen Ansatz, bei dem zunächst topische Medikamente verabreicht werden und bei ungenügender Wirksamkeit auf die Phototherapie und schliesslich auf die systemische Therapie übergegangen wird. Für die systemische Therapie der mittelschweren bis schweren Psoriasis werden Retinoide, Kortikosteroide, Methotrexat und Cyclosporin eingesetzt, die jedoch oftmals eine nur beschränkte Wirksamkeit besitzen. So zeigte eine Umfrage bei Patienten mit schwerer Psoriasis, dass die systemische Therapie in 78\% der Fälle als frustrierend und in 32\% der Fälle als zu wenig aggressiv empfunden wurde. Neben der ungenügenden Wirksamkeit wird die traditionelle systemische Langzeittherapie wegen der mangelnden Spezifität der verwendeten Präparate vielfach durch eine erhebliche Toxizität beschränkt, weshalb die Behandlung in der Regel nach Abklingen der Symptome abgebrochen wird. Da aber die meisten Patienten bereits nach kurzer Zeit ein Rezidiv erleiden, werden dringend neue Ansätze zur Langzeitkontrolle der Psoriasis benötigt. Seit einiger Zeit stehen nun biologische Wirkstoffe zur 


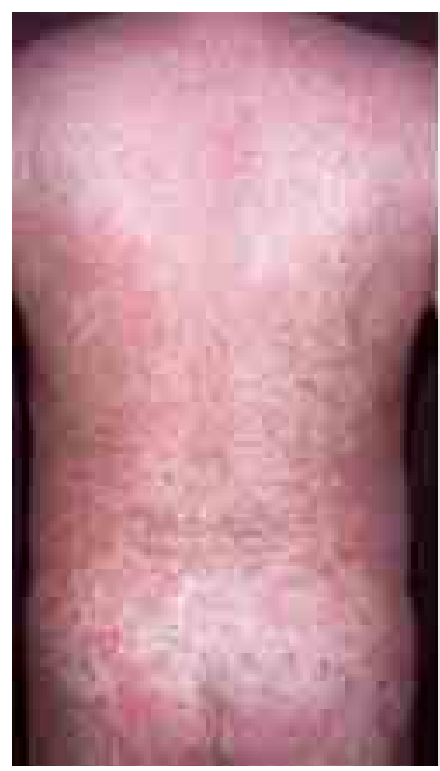

Abb. 1. a Raptiva-

Monotherapie: Monat 0.

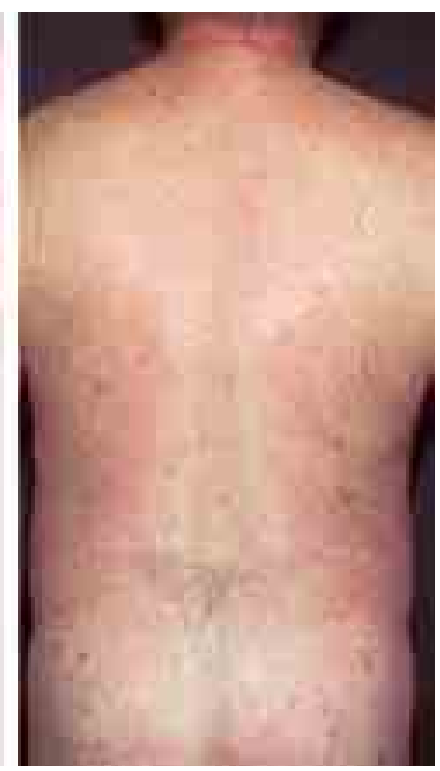

b Monat 2.

Verfügung, die einen entscheidenden Fortschritt bei der Behandlung der mittelschweren bis schweren Psoriasis erwarten lassen.

Mit dieser Erweiterung des therapeutischen Arsenals sollte nun anstelle des bisherigen stufenweisen Behandlungsansatzes eine individualisierte Therapie durchgeführt werden, die unter Berücksichtigung des Schweregrads und der Ausdehnung der Erkrankung sowie der subjektiven Bedürfnisse auf den einzelnen Patienten abgestimmt werden kann.

\section{Immunpathophysiologie der Psoriasis}

PD Dr. Nikhil Yawalkar, Bern (Schweiz), führte aus, dass die Psoriasis eine multifaktorielle Erkrankung ist, die bei genetisch prädisponierten Personen durch eine Vielzahl von Faktoren ausgelöst werden kann. Diese umfassen bakterielle Infektionen, die Einnahme gewisser Medikamente, übermässigen Alkoholkonsum oder psychischen Stress. Nach dem heutigen Verständnis der Pathogenese der Psoriasis liegt der Erkrankung wahrscheinlich eine Autoimmunreaktion zugrunde, die durch fehlgeleitete T-Lymphozyten vermittelt wird. Im Rahmen dieser Autoimmunreaktion wird ein bisher nicht näher identifiziertes Antigen von bestimmten Zellen in der Haut aufgenommen, welche das Antigen an ihrer Oberfläche präsentieren. Anschliessend wandern diese antigenpräsentierenden Zellen über die Lymphbahnen der Haut in die Lymphknoten, wo das präsentierte Antigen von den T-Lymphozyten über den «Major Histocompatibility Complex» und den «T-Cell Receptor» erkannt wird. Als Folge der Antigenpräsentation kommt es durch die Wechselwirkung von spezifischen Oberflächenmolekülen der beteiligten Zellen zu einer Aktivierung und Proliferation von T-Lymphozyten, die über den Blutkreislauf wieder in die Dermis und Epidermis gelangen. In der Haut findet eine antigenspezifische Reaktivierung dieser Gedächtnis-Ef-
fektor-T-Lymphozyten statt, was zur Ausschüttung von $\alpha$-Interferon und Tumornekrosefaktor $\alpha$ führt. Diese freigesetzten Zytokine rufen eine Entzündungsreaktion sowie eine abnorme Proliferation und Differenzierung der Keratinozyten hervor, wodurch die charakteristischen psoriatischen Plaques entstehen (Abb. 1). Das bessere Verständnis der Pathophysiologie der Psoriasis hat zur Entwicklung von Biologika geführt, welche die Aktivierung der T-Lymphozyten hemmen oder die aktivierten T-Lymphozyten selektiv zerstören oder aber die beteiligten Zytokine gezielt blockieren.

\section{Neue Behandlungsansätze}

Nach Prof. Christopher Griffiths, Manchester (GB), sind mittlerweile die Resultate von mehreren klinischen Studien veröffentlicht worden, in welchen der therapeutische Nutzen von vier verschiedenen Biologika bei der Behandlung der Psoriasis untersucht wurde. Das rekombinante Fusionsprotein Alefacept eliminiert Gedächtnis-Effektor-T-Lymphozyten. Der humanisierte monoklonale Antikörper Efalizumab hemmt die Aktivierung, Migration und Reaktivierung der T-Lymphozyten.

Zusätzlich zu den beiden auf die T-Lymphozyten wirkenden Präparaten wurden zwei weitere Biologika (Etanercept und Infliximab) in klinischen Studien untersucht, deren Wirksamkeit auf der Blockierung des Tumornekrosefaktors $\alpha$ beruht.

Aufgrund der Resultate der bislang durchgeführten klinischen Studien scheinen die Biologika eine wirksame Kontrolle der Psoriasis zu erlauben, so dass diese Präparate insbesondere bei schweren Krankheitsformen eine wertvolle Erweiterung der therapeutischen Möglichkeiten bieten. Da es sich bei der Psoriasis um eine chronische Erkrankung mit einem schubförmigen Verlauf handelt, liegt das Ziel der Behandlung nicht nur im Erreichen einer Remission, sondern insbesondere auch in deren Langzeiterhaltung.

\section{Langzeiterfahrung mit Efalizumab}

Dr. Kim Papp, Ontario (Kanada) wies darauf hin, dass mittlerweile über 2700 Psoriasispatienten im Rahmen von klinischen Studien während bis zu 2 Jahren mit Efalizumab behandelt worden sind. Zu Efalizumab liegen heute Daten sowohl über die Therapiedauer von 12 und 24 Wochen als auch über 24 Monate vor (Tab. 1).

Tabelle 1. PASI-Werte von mit Efalizumab behandelten Patienten

\begin{tabular}{|c|c|c|c|}
\hline & 12 Wochen $^{1}$ & 24 Wochen ${ }^{2}$ & 24 Monate $^{3}$ \\
\hline PASI 50 & $59 \%$ & $67 \%$ & \\
\hline PASI 75 & $27 \%$ & $44 \%$ & $68 \%$ \\
\hline PASI 90 & & & $36 \%$ \\
\hline \multicolumn{4}{|c|}{$\begin{array}{l}1 \text { Plazebokontrolliert. } \\
2 \text { Open label. } \\
{ }^{3} \text { Open label, As-treated-Analyse. }\end{array}$} \\
\hline
\end{tabular}


Die Behandlung mit Efalizumab erwies sich als gut verträglich, und bei den Patienten der Efalizumab-Gruppe wurde im Vergleich zu denjenigen der Plazebo-Gruppe auch über 24 Monate Dauertherapie keine erhöhte Inzidenz von Infektionen oder Malignitäten festgestellt.

Mit der Entwicklung der Biologika konnte bei der Behandlung der Psoriasis ein entscheidender Fortschritt erzielt werden, da diese Präparate selbst bei Patienten mit schweren Krankheitsformen vielfach zu einem deutlichen Rückgang der Symptome führen. Ausserdem weisen die Resultate von ersten Langzeitstudien darauf hin, dass die Biologika neben der schnellen Induktion der Remission auch eine dauerhafte Symptomkontrolle erlauben.

Dr. Stéphane Kuenzli

Hôpital cantonal

Clinique de dermatologie

Rue Micheli-du-Crest 24

CH-1205 Genève

Tel. 0223729423

Fax 0223729470

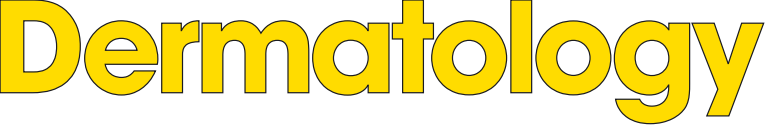

Dermatology Psychosomatics Dermatologie
$\uplus$ Psychosomatilk Dermatology

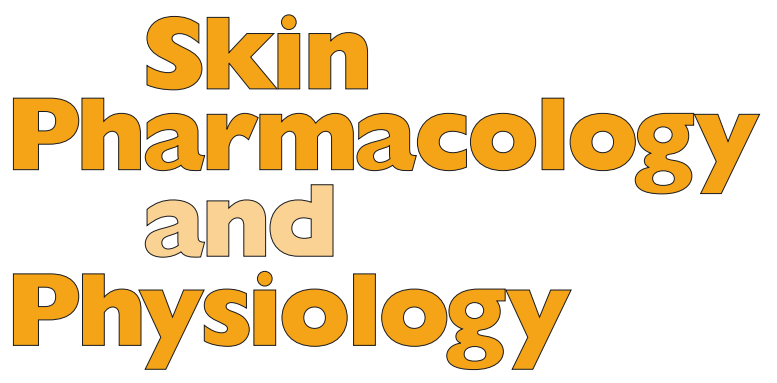

Dermatology publications that get beneath the surface of important issues

Free sample copies available online

\section{KARGER}

